Interfaces and Free Boundaries 13 (2011), 423-435

DOI $10.4171 / \mathrm{IFB} / 266$

\title{
On the blow-up mechanism of moving boundary problems
}

\author{
FRIEDRICH-MATTHIAS LIPPOTH \\ Institute of Applied Mathematics, Leibniz University of Hanover, \\ Welfengarten 1, D-30167 Hanover, Germany \\ E-mail: lippoth@ifam.uni-hannover.de
}

[Received 19 April 2010 and in revised form 3 January 2011]

\begin{abstract}
We introduce the notion of maximal solutions of a class of moving boundary problems in the sense that we characterize what inhibits global existence of solutions. The technique is demonstrated on a model describing the growth of an avascular tumor. Further applications of the main result will be given elsewhere.
\end{abstract}

Keywords: Moving boundary problem; blow-up.

\section{Introduction}

A major difficulty in the study of moving boundary problems is the understanding of their dynamical behavior 'reasonably far' from the initial geometry. In order to solve moving boundary problems, it is often needed to transform them to a pde system on a fixed reference domain involving an evolution equation for a function describing the boundary of the unknown time-dependent domains. Unfortunately, these transformations only work inside a small neighborhood of the initial domain, so that a solution can a priori only exist until it reaches the boundary of that neighborhood. Thus, taking the initial geometry close to a stable equilibrium may be the only way to guarantee that the corresponding solution will describe some 'observably dynamic behavior' for a reasonably long time.

In this paper we extend the notion of maximal solutions to a class of moving boundary problems. For a moving boundary problem we think of a blow-up scenario as a real blow-up of some physical quantity involved or as the ocurrence of a singularity in the moving boundary. Our technique is applied to the following problem:

$$
\begin{cases}-\Delta p=f(v) & \text { in } \Omega(t), \\ \partial_{t} v-\Delta v=-h(v) & \text { in } \Omega(t), \\ V=-\partial_{v} p & \text { on } \Gamma(t), \\ p=\gamma H & \text { on } \Gamma(t), \\ v=\psi & \text { on } \Gamma(t),\end{cases}
$$

where $\gamma>0$. It is complemented by the initial conditions $v(0, \cdot)=v_{0}, \Gamma(0)=\Gamma_{0}$, where we set $\Gamma(t):=\partial \Omega(t)$. The scalar system (1.1) is a model describing the growth of an avascular tumor: $\Omega(t)$ is the domain occupied by the tumor at time $t$. By $p$ we denote the cell pressure and $v$ describes the concentration of a nutrient, for example glucose, diffusing through the tumor. On the free boundary this concentration is given by the smooth function $\psi$, typically a positive constant. The normal velocity of the family $\{\Gamma(t)\}$ and the mean curvature of the surface $\Gamma(t)$ are denoted by $V$ and $H=H(t)$, respectively. The functions $h$ and $f$ are known: they describe the 
rate of consumption of the nutrient and that of the cell-proliferation. This model was introduced by Greenspan in [Gr1956] and [Gr1972] (see also [ByCh96] for more information).

Some work concerning model (1.1) has already been done: Mostly a radially symmetric setting has been investigated in [FR1999], [FR2001a], [FR2001b] and [EsMa10]. In fact, in [EsMa10] radially symmetric solutions of a model similar to (1.1) are considered. It is shown that in the case of a critical rate of cell death the tumor must vanish. Corollary 1.3 of the present article is of similar nature, because a constant rate of cell death is assumed. In this generality, a blow-up of the pressure gradient at the boundary cannot be excluded.

Finally, the papers [BF2003] and [Es2004] deal with existence of nonsymmetric solutions. In [Es2004] the existence of smooth local solutions has been proved for a large class of initial data.

At the end of this introduction we state the main theorem of this paper as well as an associated corollary. In Section 2 we first define all our notation and then discuss some helpful material from differential geometry. Section 3 deals with a survey of the problem's transformation to a fixed reference domain, and with the proofs of Theorem 1.2 and Corollary 1.3.

Let us now make some general assumptions which we keep fixed afterwards:

- $n \geqslant 2, q>n+1$ and $\beta>1-1 / q$;

- $(f, h, \psi) \in C^{\infty}(\mathbb{R}) \times C^{\infty}(\mathbb{R}) \times C^{\infty}\left(\mathbb{R}^{n}\right)$.

Definition 1.1 Let $\Omega_{0}$ be a domain in $\mathbb{R}^{n}$ of class $c^{3+\beta}$ and $v_{0}-\psi \in W_{q, 0}^{2}\left(\Omega_{0}\right)$. A classical solution of problem $(1.1)$ is a triple $(v(t, x), p(t, x), \Gamma(t))$ defined on a nontrivial interval $J:=$ $[0, S)$ such that

(i) $\bigcup_{t \in j}(\{t\} \times \Gamma(t))$ is a smooth submanifold of $\mathbb{R}^{n+1}$;

(ii) $\{\Gamma(t): t \in J\}$ is of class $(m b)^{(3, \beta)}$;

(iii) $v \in B U C^{\infty}\left(\bigcup_{t \in(\varepsilon, S-\varepsilon)}(\{t\} \times \Omega(t))\right.$, $\left.\mathbb{R}\right)$ for all $0<\varepsilon<S / 2$;

(iv) $p(t, \cdot) \in W_{q}^{2}(\Omega(t))$ and $v \circ \Theta^{t} \in C\left(\left[0, \delta^{t}\right), W_{q}^{2}\left(D^{t}\right)\right)$ for $t \in J$;

(v) $(v, p, \Gamma)$ satisfy the equations (1.1) pointwise on $\bigcup_{t \in J}(\{t\} \times \bar{\Omega}(t))$.

The class $(m b)^{(k, \alpha)}$ is a convenient tool to measure the spatial regularity of a family of hypersurfaces in $\mathbb{R}^{n+1}$. The precise definition of this class and of the mapping $\Theta^{t}$ in (iv) is provided in Section 2. Notice that (iv) in particular implies that $v(t) \in W_{q}^{2}(\Omega(t))$ for $t \in J$.

A classical solution is maximal if there is no proper extension of it. In this case, $t^{+}$denotes the maximal time of existence.

THEOREM 1.2 Let $\Omega_{0}$ be a domain in $\mathbb{R}^{n}$ of class $c^{3+\beta}$ and $v_{0}-\psi \in W_{q, 0}^{2}\left(\Omega_{0}\right)$. Then there exists a unique maximal classical solution of problem (1.1). If $t^{+}<\infty$, then there exists a sequence $t_{n} \rightarrow t^{+}$such that

- $\left\|v\left(t_{n}\right)\right\|_{B U C^{1}\left(\Omega\left(t_{n}\right)\right)}+\left\|p\left(t_{n}\right)\right\|_{B U C^{1}\left(\Omega\left(t_{n}\right)\right)} \rightarrow \infty$ as $t_{n} \rightarrow t^{+}$, or

- $A\left(\Gamma\left(t_{n}\right)\right) \rightarrow 0$ as $t_{n} \rightarrow t^{+}$.

The quantity $A(\Gamma)$ measures the maximal possible size of spheres which intersect $\Gamma$ only in one point (see Section 2). Therefore, the second condition covers the situation where different regions of the tumor grow together, mathematically speaking the occurrence of self-intersection. This has been the case in numerical experiments (see [CrLoNi]). The condition can also describe shrinking to a point or the development of singularities in $\Gamma(t)$ :

Corollary 1.3 Let $c>0$. Assume $v_{0} \equiv c, \psi \equiv c, h(c)=0$ and $f(c)=-\alpha_{0}<0$. Let $(v, p, \Gamma)$ be the unique classical solution of system (1.1) corresponding to some initial surface $\Gamma_{0}$. 
Then there exists a sequence $t_{n} \rightarrow t^{+}$such that

$$
A\left(\Gamma\left(t_{n}\right)\right)^{-1}+\left\|\nabla p\left(t_{n}\right)\right\|_{C\left(\Gamma\left(t_{n}\right)\right)} \rightarrow \infty \quad \text { as } t_{n} \rightarrow t^{+} .
$$

\section{Notation and helpful material}

In the following by a $C^{k+\alpha}\left[c^{k+\alpha}\right]$-domain $\Omega$ we mean a bounded connected open subset of $\mathbb{R}^{n}$ whose boundary $\Gamma:=\partial \Omega$ is a compact closed embedded hypersurface of regularity $C^{k+\alpha}\left[c^{k+\alpha}\right]$. Here $c^{k+\alpha}$ denotes the small Hölder space, that is, the closure of the (sufficiently) smooth functions in the usual Hölder norm (cf. [TrI83], [TrII92], [Lu1995]). The definition of the space $B U C^{s}$ can be found in [Es2004], [Ama93].

If $\Omega$ is a domain, we define a tubular neighborhood of $\Gamma$ to be an open set $N$ which is the image of the diffeomorphic map

$$
X: \Gamma \times(-\delta, \delta) \rightarrow \mathbb{R}^{n}, \quad(x, a) \mapsto x+a \cdot v(x),
$$

where $v(x)$ is the outer unit normal vector at $x \in \Gamma$ and $\delta>0$ is sufficiently small. We decompose the inverse of $X$ as $X^{-1}=\left(P_{[\Gamma]}, \Lambda_{[\Gamma]}\right)$, where $P_{[\Gamma]}$ is the metric projection of a point $x$ onto $\Gamma$, and $\Lambda_{[\Gamma]}$ is the signed distance function with respect to $\Gamma$. Clearly, if $\operatorname{im}(X)$ is a tubular neighborhood of $\Gamma$, so is $\operatorname{im}\left(\left.X\right|_{\Gamma \times(-a, a)}\right)$ if $a<\delta$. The set of all tubular neighborhoods of a surface $\Gamma$ is denoted by $\mathcal{T} \mathcal{N}(\Gamma)$.

We say that $\Omega \subset \mathbb{R}^{n}$ satisfies the interior sphere condition (ISC) if for any $x \in \Gamma$ there is a ball $B_{x} \subset \bar{\Omega}$ such that $\Gamma \cap B_{x}=\{x\}$; and $\Omega$ satisfies the exterior sphere condition (ESC) if its complement has ISC. For a suitable domain $\Omega$ and $x \in \Gamma$, let $R_{i}(x)\left[R_{e}(x)\right]$ be the supremum over all radii of possible interior [exterior] spheres at $x$ and define

$$
A_{i}(\Gamma):=\inf _{x \in \Gamma} R_{i}(x), \quad A_{e}(\Gamma):=\inf _{x \in \Gamma} R_{e}(x), \quad A(\Gamma):=\min \left\{A_{i}(\Gamma), A_{e}(\Gamma)\right\} .
$$

Finally, if $N \in \mathcal{T} \mathcal{N}(\Gamma)$, then $r(N):=\operatorname{dist}(\partial N, \Gamma)$ is the radius of $N$.

DEFinition 2.1 We say that a family $\left\{\Gamma_{\alpha}: \alpha \in \mathrm{A}\right\}$ of submanifolds of $\mathbb{R}^{n}$ has uniformly bounded $C^{k}$-geometry if there exist $m \in \mathbb{N}$ and $K, L>0$ such that

- for each $\alpha \in \mathrm{A}$ there is a $C^{k}$-atlas $\left(U_{\alpha}^{l}, \varphi_{\alpha}^{l}\right)$ for $\Gamma_{\alpha}$, where $1 \leqslant l \leqslant m$;

- if $\varphi_{\alpha}^{l} \in \operatorname{diff}^{k}\left(V_{\alpha}^{l}\right.$, $\left.W_{\alpha}^{l}\right)$ (i.e. $\left.U_{\alpha}^{l}=\left(\varphi_{\alpha}^{l}\right)^{-1}\left[W_{\alpha}^{l}\right] \cap \Gamma_{\alpha}\right)$, then, for some $x_{\alpha}^{l} \in U_{\alpha}^{l},\left\|\varphi_{\alpha}^{l}\right\|_{C^{k}\left(V_{\alpha}^{l}\right)}+$ $\left\|\left(\varphi_{\alpha}^{l}\right)^{-1}-x_{\alpha}^{l}\right\|_{C^{k}\left(W_{\alpha}^{l}\right)} \leqslant K$ for $(l, \alpha) \in\{1, \ldots, m\} \times \mathrm{A}$;

- for each $\alpha \in \mathrm{A}$ there is a partition of unity $\left\{\pi_{\alpha}^{l}\right\}, 1 \leqslant l \leqslant m$, subordinate to the above covering, such that $\left\|\pi_{\alpha}^{l} \circ\left(\varphi_{\alpha}^{l}\right)^{-1}\right\|_{C^{k}\left(W_{\alpha}^{l}\right)} \leqslant L$ for $(l, \alpha) \in\{1, \ldots, m\} \times \mathrm{A}$.

Lemma 2.2 Let $\mu>0$ be given, let $\left\{\Omega_{\alpha}: \alpha \in \mathrm{A}\right\}$ be a family of $C^{2}$-domains in $\mathbb{R}^{n}$, and let $\Gamma_{\alpha}$ be the boundary of $\Omega_{\alpha}$. Suppose

$$
\inf _{\alpha \in \mathrm{A}} A\left(\Gamma_{\alpha}\right) \geqslant \mu .
$$

Then for each $\alpha \in \mathrm{A}$ there is $N_{\alpha} \in \mathcal{T} \mathcal{N}\left(\Gamma_{\alpha}\right)$ such that $\inf _{\alpha \in \mathrm{A}} r\left(N_{\alpha}\right) \geqslant \mu$. Moreover, if $N \in$ $\mathcal{T} \mathcal{N}\left(\Gamma_{\alpha}\right)$ satisfies $r(N) \leqslant a<\mu$, then the quantity

$$
\sup _{\Gamma_{\beta} \subset N}\left\|\Lambda_{\left[\Gamma_{\alpha}\right]}\right\|_{C^{2}\left(\Gamma_{\beta}\right)}
$$

can be estimated from above in terms of the numbers $a$ and $\mu$. Finally, if $\sup _{\alpha \in \mathrm{A}} \operatorname{area}\left(\Gamma_{\alpha}\right)<\infty$, then $\left\{\Gamma_{\alpha}\right\}$ has uniformly bounded $C^{2}$-geometry. Here area $(\Gamma):=\int_{\Gamma} 1$. 
Proof. The first statement can be easily seen by a careful reading of Chapter 14.6 in [GilTru]. For the second one we notice

$$
\sup _{\Gamma_{\beta} \subset N}\left\|\Lambda_{\left[\Gamma_{\alpha}\right]}\right\|_{C\left(\Gamma_{\beta}\right)} \leqslant r(N) \leqslant a .
$$

Let $\kappa^{1}\left(x_{\alpha}\right), \ldots, \kappa^{n-1}\left(x_{\alpha}\right)$ be the principal curvatures of the surface $\Gamma_{\alpha}$ at $x_{\alpha} \in \Gamma_{\alpha}$. Inside $N$ we have

$$
\nabla\left(\Lambda_{\left[\Gamma_{\alpha}\right]}\right)(x)=v\left(z_{\alpha}\right)
$$

where $z_{\alpha}:=P_{\left[\Gamma_{\alpha}\right]}(x)$, as well as

$$
D^{2}\left(\Lambda_{\left[\Gamma_{\alpha}\right]}\right)(x)=\operatorname{diag}\left[\frac{-\kappa^{1}\left(z_{\alpha}\right)}{1-\kappa^{1}\left(z_{\alpha}\right) \Lambda_{\left[\Gamma_{\alpha}\right]}(x)}, \ldots, \frac{-\kappa^{n-1}\left(z_{\alpha}\right)}{1-\kappa^{n-1}\left(z_{\alpha}\right) \Lambda_{\left[\Gamma_{\alpha}\right]}(x)}, 0\right]
$$

with respect to a principal coordinate system centered at $z_{\alpha}$, since $\left|\kappa^{i}\right| \leqslant 1 / \mu$. Thus, to prove the theorem, it suffices to construct a suitable $C^{2}$-atlas for each of the surfaces $\Gamma_{\alpha}$. We fix $\alpha \in$ A. Without loss of generality we may work near $0 \in \mathbb{R}^{n}$, which is assumed to be an element of $\Gamma_{\alpha}$.

Let $\Gamma_{\alpha}$ be locally the graph of some $C^{2}$-function $g$ satisfying $g(0)=0, \nabla g(0)=0$, and let $\psi(y):=(y, g(y))$. Fix a principal coordinate system (which is an orthonormal basis) $B:=B_{p}:=$ $\left\{v_{1}(x), \ldots, v_{n-1}(x)\right\}$ centered at $p=\psi(x) \in \Gamma_{\alpha}$ and let $B^{\prime}:=\left\{\partial_{1} \psi(x), \ldots, \partial_{n-1} \psi(x)\right\}$. Let $I I_{C}:=I I_{C}(p)$ be the representation matrix of the second fundamental form $I I(p)$ with respect to $C \in\left\{B, B^{\prime}\right\}$. If $\operatorname{cor}_{C}(z)$ is the coordinate vector of $z \in T_{p} \Gamma_{\alpha}$ with respect to $C \in\left\{B, B^{\prime}\right\}$, it follows from basic linear algebra that

$$
\frac{D^{2} g(x)}{\sqrt{1+|\nabla g(x)|^{2}}}=I I_{B^{\prime}}=\left(M_{B}^{B^{\prime}}\right)^{t} I I_{B} M_{B}^{B^{\prime}}=\left(M_{B}^{B^{\prime}}\right)^{t} \operatorname{diag}\left[\kappa_{1}, \ldots, \kappa_{n-1}\right] M_{B}^{B^{\prime}},
$$

where $M_{B}^{B^{\prime}} \operatorname{cor}_{B^{\prime}}(z)=\operatorname{cor}_{B}(z)$ defines the action of $M_{B}^{B^{\prime}}$. Moreover, since $B$ is an orthonormal basis, we have

$$
\left(M_{B}^{B^{\prime}}\right)^{t} M_{B}^{B^{\prime}}=\left(\partial_{i} \psi(x) \cdot \partial_{j} \psi(x)\right)_{i, j}=I+\nabla g(x)(\nabla g(x))^{t},
$$

provided the evaluations of $g$ and its partial derivatives at $x$ are well defined, of course. Keep in mind that $\left|\kappa^{i}\right| \leqslant 1 / \mu$. Let $\mathcal{V}$ be the largest connected open set where $g$ can be defined. It is not difficult to see that $|\nabla g(x)| \rightarrow \infty$ as $x \rightarrow \partial \mathcal{V}$. Let

$$
W:=\bigcup\left\{U \in \mathcal{U}(0) \cap \mathcal{V}:\|\nabla g\|_{C(U)} \leqslant 1\right\} \cap \mathbb{B}(0,1) .
$$

Here, $\mathcal{U}(0)$ denotes the neighborhood filter of $0 \in \mathbb{R}^{n-1}$. We conclude

$$
\left\|D^{2} g\right\|_{\infty, W} \leqslant(\sqrt{2} N / \mu)\left\|M_{B}^{B^{\prime}}\right\|_{\infty, W}^{2} \leqslant 2 \sqrt{2} N / \mu
$$

where $N:=(n-1)^{2}$. Let $y \in \partial W$ be given. Then, if $|y|<1$, it follows from the last inequality and the mean value theorem that $|y| \geqslant \mu /(2 \sqrt{2(n-1)} N)$. Moreover, $\|g\|_{C^{2}(W)} \leqslant \max \{1,2 \sqrt{2} N / \mu\}$. Since this estimate is independent of $\alpha \in A$, it remains to construct a suitable family $\pi_{\alpha}^{l}$ of partitions of unity, subordinate to the sets $g[W]$. This is possible because of the lower bound for the size of $W$. To prove the last statement, we fix an open real interval $J$ containing 0 and define local coordinates by

$$
\varphi^{-1}: W \times J \rightarrow \mathbb{R}^{n}, \quad(x, y) \mapsto(x, y+g(x)) .
$$


Clearly, $\varphi(x, y)=(x, y-g(x))$ for $(x, y) \in \varphi^{-1}[W \times J] \subset \mathbb{R}^{n}$. Moreover, if $\sup _{\alpha \in \mathrm{A}}$ area $\left(\Gamma_{\alpha}\right)$ $<\infty$, we can cover each surface $\Gamma_{\alpha}$ by, say, $m \in \mathbb{N}$ charts constructed as above, because, if not, there would be a sequence $\left(\alpha_{n}\right) \subset \mathrm{A}$ such that

$$
\int_{\Gamma_{\alpha_{n}}} 1=\sum_{l=1}^{a_{\alpha_{n}}(n)} \int_{\operatorname{supp}\left(\pi_{\alpha_{n}}^{l}\right)} \pi_{\alpha_{n}}^{l} \geqslant a_{\alpha_{n}}(n) \cdot C,
$$

where $a_{\alpha_{n}}(n) \geqslant n$ and $C=C(\min \{1, \mu /(2 \sqrt{2(n-1)} N))$. This completes the proof.

Let $k \in \mathbb{N}$ and $\alpha \in(0,1)$. Given any compact manifold $M$ of class $C^{k}$, let $\mathbb{B}_{\varepsilon}^{k}(M)$ be the open ball in $C^{k}(M)$ with radius $\varepsilon>0$ and center 0 . The next theorem ensures that any hypersurface $M$ of class $c^{k+\alpha}$ with $k \geqslant 2$ can always be represented as the graph of a $c^{k+\alpha}$-function in the normal direction of an analytic surface. The proof is based on a level set approximation of $M$ and the implicit function theorem. The author wants to emphasize that this idea is due to M. Bergner. In order to avoid too much technicalities we omit a precise proof here. The details are given in [BEL2011].

THEOREM 2.3 Let $\Omega$ be a $C^{k+\alpha}\left[c^{k+\alpha}\right]$-domain in $\mathbb{R}^{n}, k \geqslant 2$, and let $\varepsilon>0$ be given. Then there exists

- a domain $D$ whose boundary $\Sigma$ is an analytic embedded hypersurface;

- a tubular neighborhood $N$ of $\Sigma$ containing $\partial \Omega$;

- a function $\rho \in C^{k+\alpha}(\Sigma)\left[\rho \in c^{k+\alpha}(\Sigma)\right]$;

- a norm on $C^{1}(\Sigma)$

such that the map

$$
\theta_{\rho}: \Sigma \rightarrow \partial \Omega, \quad x \mapsto x+\rho(x) \cdot v(x),
$$

is a $C^{k+\alpha}\left[c^{k+\alpha}\right]$-diffeomorphism and $\rho \in \mathbb{B}_{\varepsilon}^{1}(\Sigma)$.

To the best knowledge of the author, up to now a rigorous proof of the statement of Theorem 2.3 has not been published, although it is a frequently made assumption in many articles.

In the special situation of this article, we will need Theorem 2.3 only to represent the initial surface $\Gamma_{0}$. It becomes more important, of course, if one tries to continue a flow which does not produce smooth surfaces immediately after the initial time.

However, it is now natural to introduce the classes $(M B)^{(k, \alpha)}$ :

Definition 2.4 Let $J$ be a real interval. The family $\{\Gamma(t): t \in J\}$ is of class $(M B)^{(k, \alpha)}$ if, given $t_{0} \in J \backslash \sup J$, there is a smooth manifold $\Sigma=\Sigma\left(t_{0}\right)$, a positive number $\delta=\delta\left(t_{0}\right)$, and a function

$$
\rho=\rho\left(t_{0}\right) \in C\left([0, \delta), C^{k+\alpha}(\Sigma)\right) \cap C^{1}([0, \delta), C(\Sigma))
$$

such that $\Gamma\left(t_{0}+h\right)=\theta_{\rho(h)}[\Sigma]$ for $h \in[0, \delta)$. It is of class $(m b)^{(k, \alpha)}$ if this holds true for the little Hölder spaces $c^{k+\alpha}$. We shall use the notation $\Sigma^{t_{0}}:=\Sigma\left(t_{0}\right), \delta^{t_{0}}:=\delta\left(t_{0}\right)$.

The abbreviation 'MB' should remind of 'moving boundary'. If $\{\Gamma(t)\}$ is of class $(M B)^{(k, \alpha)}$ $\left[(m b)^{(k, \alpha)}\right]$, we use the symbols $D^{t_{0}}$ and $\Theta^{t_{0}}$ to denote the domain enclosed by $\Sigma^{t_{0}}$ (cf. Theorem 2.3) and the family of diffeomorphisms $\theta_{\rho\left(t_{0}\right)(\cdot)}:\left[0, \delta^{t_{0}}\right) \rightarrow \operatorname{Abb}\left(\mathbb{R}^{n}, \mathbb{R}^{n}\right)$, i.e. $\Theta^{t_{0}}(h) \in$ $\operatorname{diff}\left(\bar{D}^{t_{0}}, \bar{\Omega}\left(t_{0}+h\right)\right), h \in\left[0, \delta^{t_{0}}\right)$. 


\section{Time localizations}

Let the data of the problem be as described in Section 1 and fix a smooth manifold $\Sigma$, a tubular neighborhood $N$ of $\Sigma$ containing $\partial \Omega$, and a function $\rho_{0} \in c^{3+\beta}(\Sigma)$ such that $\theta_{\rho_{0}}$ is a $c^{3+\beta}$ diffeomorphism from $\Sigma$ onto $\Gamma_{0}$. If $a:=r(N)$, by Lemma 2.2 we can assume that $\left\|\rho_{0}\right\|_{C^{1}(\Sigma)}<$ $a / 5$. Notice that $\rho_{0} \in B_{q q}^{4-1 / q}(\Sigma)$ thanks to $\beta>1-1 / q$, thus $c^{3+\beta}(\Sigma) \hookrightarrow B_{q q}^{4-1 / q}(\Sigma)$.

The assumption that the initial surface $\Gamma_{0}$ should be of class $c^{3+\beta}$ instead of $B_{q q}^{4-1 / q}$ was made due to the fact that the representation theorem 2.3 treats manifolds whose smoothness is measured in the Hölder scale. Thus, the existence of a smooth reference surface $\Sigma$ is not an assumption but a fact, provided $\Gamma_{0}$ is of class $c^{3+\beta}$. Inside $N$ the diffeomorphisms $\theta_{\rho(\text { (.) }}$ transform problem (1.1) into

$$
\begin{cases}A(\rho) r=f(w) & \text { in } J \times D, \\ \partial_{t} w+A(\rho) w=R(w, r, \rho)-h(w) & \text { in } J \times D, \\ \partial_{t} \rho+B(\rho) r=0 & \text { on } J \times \Sigma, \\ r=H(\rho) & \text { on } J \times \Sigma, \\ w=\chi(\rho) & \text { on } J \times \Sigma, \\ w(0, \cdot)=w_{0} & \text { in } D, \\ \rho(0, \cdot)=\rho_{0} & \text { in } \Sigma\end{cases}
$$

where we set $w_{0}:=v_{0} \circ \theta_{\rho_{0}}$ and $\chi(\rho)=\psi \circ \theta_{\rho}$. The term $R$ arises from the transformation of the time derivative $v_{t}$. It is worth mentioning here that it is possible to extend $\theta_{\rho(t)}$ to a diffeomorphism $\tilde{\theta}_{\rho(t)} \in \operatorname{diff}^{3+\beta}\left(\mathbb{R}^{n}, \mathbb{R}^{n}\right) \cap \operatorname{diff}^{3+\beta}(D, \Omega(t))$. Our notation does not distinguish between $\theta$ and $\tilde{\theta}$. All necessary calculations and notation can be found in [Es2004].

Now, if

$$
r(w, \rho):=S(\rho) f(w)+T(\rho) H(\rho),
$$

i.e. $r(w, \rho)$ is the unique solution of the elliptic problem

$$
\begin{cases}A(\rho) r=f(w) & \text { in } D \\ r=H(\rho) & \text { on } \Sigma\end{cases}
$$

we get

$$
\begin{cases}\partial_{t} w+A(\rho) w=R(w, r(w, \rho), \rho)-h(w) & \text { in } j \times D, \\ w=\chi(\rho) & \text { on } J \times \Sigma, \\ \partial_{t} \rho+B(\rho) T(\rho) H(\rho)=B(\rho) S(\rho) f(w) & \text { on } j \times \Sigma, \\ w(0, \cdot)=w_{0} & \text { in } D, \\ \rho(0, \cdot)=\rho_{0} & \text { in } \Sigma .\end{cases}
$$

Letting $u:=w-\chi(\rho)$ we have

$$
\begin{cases}\partial_{t} u+A(\rho) u=R\left(u_{\chi}, r\left(u_{\chi}, \rho\right), \rho\right)-h\left(u_{\chi}\right) & \\ \quad-A(\rho) \chi(\rho)-Q\left(r\left(u_{\chi}, \rho\right), \rho\right) & \text { in } \dot{J} \times D, \\ u=0 & \text { on } J \times \Sigma, \\ \partial_{t} \rho+B(\rho) T(\rho) H(\rho)=B(\rho) S(\rho) f\left(u_{\chi}\right) & \text { on } \dot{J} \times \Sigma, \\ u(0, \cdot)=w_{0}-\chi\left(\rho_{0}\right) & \text { in } D, \\ \rho(0, \cdot)=\rho_{0} & \text { in } \Sigma,\end{cases}
$$

where we have used the notation $u_{\chi}:=u_{\chi}(\rho):=u+\chi(\rho)$. Here $Q$ results from the differentiation 
of the transformed boundary data $\chi(\rho)$ with respect to time. We consider (3.4) as the system

$$
\left\{\begin{array}{cc}
\partial_{t} u+A(\rho) u=R\left(u_{\chi}, r\left(u_{\chi}, \rho\right), \rho\right)-h\left(u_{\chi}\right) & \\
-A(\rho) \chi(\rho)-Q\left(r\left(u_{\chi}, \rho\right), \rho\right) & \text { in } \dot{J} \times D, \\
\partial_{t} \rho+B(\rho) T(\rho) H(\rho)=B(\rho) S(\rho) f\left(u_{\chi}\right) & \text { on } \dot{J} \times \Sigma, \\
u(0, \cdot)=w_{0}-\chi\left(\rho_{0}\right) & \text { in } D, \\
\rho(0, \cdot)=\rho_{0} & \text { in } \Sigma,
\end{array}\right.
$$

over a suitable function space including the zero boundary condition. Finally, we split the mean curvature $H(\rho)$ into a leading quasilinear part $P(\rho) \rho$ and a nonlinear part $Q(\rho)$ containing only first order terms: $H(\rho)=P(\rho) \rho+Q(\rho)$ (cf. Lemma 3.1 in [EsSi97a] and Lemma 3.2 in [Es2004]). We arrive at

$$
\begin{cases}\partial_{t} u+A(\rho) u=F(u, \rho) & \text { in } \dot{j} \times D, \\ \partial_{t} \rho+B(\rho) T(\rho) P(\rho) \rho=G(u, \rho) & \text { on } \dot{J} \times \Sigma, \\ u(0, \cdot)=w_{0}-\chi\left(\rho_{0}\right) & \text { in } D, \\ \rho(0, \cdot)=\rho_{0} & \text { in } \Sigma,\end{cases}
$$

where

$$
\begin{aligned}
& F(u, \rho):=R\left(u_{\chi}, r\left(u_{\chi}, \rho\right), \rho\right)-h\left(u_{\chi}\right)-A(\rho) \chi(\rho)-Q\left(r\left(u_{\chi}, \rho\right), \rho\right), \\
& G(u, \rho):=-B(\rho)\left[T(\rho) K(\rho)-S(\rho) f\left(u_{\chi}\right)\right] .
\end{aligned}
$$

The mappings $A, B T P, F$ and $G$ are smooth between various spaces. The precise mapping properties of $A$ and $B T P$ can be found in Lemma 2.2 and Theorem 4.1 of [EsSi97a], and in Lemmas 3.1 and 4.4 of [Es2004]. The nonlinearity $(F, G)$ (in the case $h(y)=y)$ is investigated in Lemma 4.6 of [Es2004].

We choose $b:=\max \left\{2\left\|\rho_{0}\right\|_{C^{1+\beta}(\Sigma)}, a / 5\right\}$ and define

$$
\mathcal{U}:=H_{q, 0}^{2}(D) \times\left\{\rho \in B_{q q}^{4-1 / q}(\Sigma):\|\rho\|_{c^{1+\beta}(\Sigma)}<b,\|\rho\|_{c^{1}(\Sigma)}<a / 5\right\},
$$

abbreviated by $\mathcal{U}:=H_{q, 0}^{2}(D) \times \mathcal{A}$. Then $\mathcal{U}$ is an open subset of $\mathbb{D}:=H_{q, 0}^{2}(D) \times B_{q q}^{4-1 / q}(\Sigma)$ and it contains $\left(u_{0}, \rho_{0}\right) . H_{q}^{s}$ and $B_{q q}^{s}$ denote the Bessel potential and Besov spaces, respectively (see [TrI83], [TrII92]). Now, in literally the same way as in [Es2004] and using the fact that $h$ induces smooth Nemytskii operators acting on various function spaces, one can show the existence of a unique solution of (3.6), that is, a pair of functions $(u, \rho)$ defined on a nontrivial interval $J:=[0, T)$ such that

- $\rho \in C\left(J, B_{q q}^{4-1 / q}(\Sigma) \cap \mathcal{A}\right) \cap C^{1}\left(J, B_{q q}^{1-1 / q}(\Sigma)\right) \cap C^{\infty}\left(\dot{J}, B_{q q}^{4+k-1 / q}(\Sigma)\right)$ for all $k \in \mathbb{N}$, - $u \in C\left(J, H_{q}^{2}(D)\right) \cap C^{1}\left(J, L_{q}(D)\right) \cap C^{\infty}\left(\dot{J}, H_{q}^{2+k}(D)\right)$ for all $k \in \mathbb{N}$,

- $\left.u(t)\right|_{\Sigma}=0$ for $t \in J$, and

- $(u, \rho)$ satisfy the equations in (3.6) pointwise in $J$.

Moreover, modifying Lemma 4.6 in [Es2004] in an obvious way and using the generation properties of the operator $B(\cdot) T(\cdot) P(\cdot)$ in the Hölder space setting which are stated for example in [EsSi97a] we obtain the continuity of the distance function $\rho$ at 0 with respect to the $c^{3+\beta}$ topology by standard arguments based on interpolation and the variation-of-constants formula: $\rho \in C\left(J, c^{3+\beta}(\Sigma)\right)$. Following the abstract theory (cf. Theorems 12.1 and 12.5 in [Ama93]), $T$ can be chosen maximal in the sense that

$$
\|(u(t), \rho(t))\|_{\mathbb{D}} \stackrel{t \rightarrow T}{\longrightarrow} \infty \quad \text { or } \quad(u(t), \rho(t)) \stackrel{t \rightarrow T}{\longrightarrow} \partial \mathcal{U},
$$

provided $T<\infty$. 
Notice that the transformation of problem (1.1) was made in such a way that if $(u, \rho)$ is a solution to (3.6) in the sense stated above, then

$$
(v(t), p(t), \Gamma(t)):=\left(u(t)+\chi(\rho(t)) \circ \theta_{\rho(t)}^{-1}, r(t) \circ \theta_{\rho(t)}^{-1}, \theta_{\rho(t)}[\Sigma]\right)
$$

solves (1.1), where $r=r(u, \rho)$ is defined by (3.2). Let us introduce the notion of time-local solutions:

DEFINITION 3.1 A solution $(u, \rho)$ of (3.6) corresponding to the initial value $\left(u_{0}, \rho_{0}\right)$ is said to be a time-local solution of (1.1).

It can be shown that the transformations sketched in this section can also be used to construct a time-local solution of (3.6) from a given classical solution of (1.1) in the sense of Definition 1.1. For this we have to use the regularity assumption $v \circ \Theta^{0} \in C\left(\left[0, \delta^{0}\right), W_{q}^{2}\left(D^{0}\right)\right)$ in Definition 1.1(iv). It guarantees uniqueness of classical solutions for a short time.

LEMMA 3.2 Let $(u, \rho)$ be a time-local solution of (3.6) and assume $T<\infty$. If $(u, r) \in$ $L_{\infty}\left(J, B U C^{1}(D) \times B U C^{1}(D)\right)$, then $(u, \rho)$ extends to a continuous function on $\bar{J}:=[0, T]$ with values in $H_{q}^{2}(D) \times c^{3+\beta}(\Sigma)$. Moreover, $\rho(T) \in \partial \mathcal{A}$.

Proof. By definition, $\rho$ takes its values in the set $\mathcal{A}$. Thus, $\rho$ is bounded in $c^{1+\beta}(\Sigma)$. Therefore, the set $\{\rho(t) \mid t \in[0, T)\}$ is relatively compact in $c^{1+\beta^{\prime}}(\Sigma)$ for $\beta^{\prime}<\beta$. Notice that the mappings $P(\cdot)$, $K(\cdot)$ and $\sigma \mapsto \theta_{\sigma}$ can be defined on the closure of $\mathcal{A}$ in the norm of $c^{1+\beta^{\prime}}(\Sigma)$. Therefore

$$
\sup _{\delta \in J}\left(\left\|\theta_{\rho(\delta)}\right\|_{C^{1}\left(\bar{D}, \mathbb{R}^{n}\right)}+\left\|\theta_{\rho(\delta)}^{-1}\right\|_{C^{1}\left(\bar{\Omega}_{\rho(\delta)}, \mathbb{R}^{n}\right)}\right)<\infty .
$$

Direct calculations (see [Kn2007]) show that from this and from our assumption on $r$ we get a bound for $B(\rho) r$ in the norm of $C(\Sigma)$, so that the velocity $\rho_{t}$ is bounded in the same norm (cf. the third equation in (3.1)). Also, from $P(\rho) \rho=r-K(\rho)$ and well-known generation properties of the operator $P(\rho)$, we are allowed to conclude $\|\rho\|_{C\left(J, c^{2+\mu}(\Sigma)\right)}<\infty$ for some $\mu>0$. Thus, interpolating between $C(\Sigma)$ and $c^{2+\mu}(\Sigma)$ and using the mean value theorem, we find that $\rho \in$ $B U C^{\varepsilon}\left(J, c^{2+\mu^{\prime}}(\Sigma)\right)$, where $\mu>\mu^{\prime}>0$ and $\varepsilon$ is a suitable positive number. Thus, our assumption on $u$ implies that $G(u, \rho)$ is bounded in $c^{\mu^{\prime}}(\Sigma)$. Since the mapping $B(\cdot) T(\cdot) P(\cdot)$ can be defined on the closure of $\mathcal{A}$ in the norm of $c^{2+\mu^{\prime}}(\Sigma)$, the second equation in (3.6) and the generation properties of $B(\rho) T(\rho) P(\rho)$ provide a variation-of-constants formula based bootstrapping procedure ending up (after two steps) with a bound for $\rho$ in the norm of $c^{3+\beta}(\Sigma)$.

Turning to the function $u$, we find that $F(u, \rho)$ is bounded in $L_{q}(D)$. Since $A(\cdot)$ can be defined on the closure of $\mathcal{A}$ in the norm of $c^{2+\mu^{\prime}}(\Sigma)$, a (two-step) bootstrapping procedure based on the variation-of-constants formula leads to a bound for $u$ in the $H_{q}^{2}(D)$-norm, so we also get a bound for the time derivative $u_{t}$ in the norm of $L_{q}(D)$. Therefore $u \in B U C^{\varepsilon^{\prime}}\left(J, H_{q}^{s}\right)$ for $s<2$ and suitable $\varepsilon^{\prime}>0$. By a bootstrapping argument we conclude that in fact $(u, \rho) \in B U C^{\varepsilon^{\prime \prime}(k)}\left([\gamma, T), H_{q}^{k}(D) \times\right.$ $c^{k}(\Sigma)$ ) for any positive $\gamma<T, k \in \mathbb{N}$ and an interpolation exponent $\varepsilon^{\prime \prime}>0$ depending on $k$. Thus, $\rho(T) \in \partial \mathcal{A}$ by (3.7).

REMARK 3.3 The a priori estimates for $\rho$ which we used in the proof of the last lemma depend highly on the geometry of $\Sigma$. As we will see later, we do not need them to be uniform with respect to that manifold in any sense. What we need to be uniform is just the property of $P(\sigma)$, $B(\sigma) T(\sigma) P(\sigma), \sigma \in \mathcal{A}$, to generate an analytic semigroup. We will comment on this in more detail at the very end of this section, after defining the necessary notation (cf. Remark 3.4). 
In order to prove our main result, we need to comment on an important relation between the original problem and its transformed version: If $(u, \rho)$ is a time-local solution to (1.1), then the surface $\Gamma(t)=\theta_{\rho(t)}[\Sigma]$ is the zero level set of the function

$$
\varphi_{\rho(t)}: N \rightarrow \mathbb{R}, \quad x \mapsto \Lambda_{[\Sigma]}(x)-\rho(t)\left(P_{[\Sigma]}(x)\right) .
$$

From this we conclude easily $V\left(t, \theta_{\rho(t)}(x)\right)=\rho_{t}(t)(x) /\left|\nabla\left(\varphi_{\rho(t)}\right)\left(\theta_{\rho(t)}(x)\right)\right|$ (see again [Es2004]). We are now prepared for

Proof of Theorem 1.2. Let the initial data of the problem be given and fix a suitable reference manifold $\Sigma$ as described in this section. The existence result for time-local solutions ensures that we can find a solution of problem (1.1) either on $[0, \infty)$ or on some finite interval $[0, T)$. Then the maximal interval of existence is

$$
\left[0, t^{+}\right):=\bigcup_{T>0}\{[0, T):(1.1) \text { has a solution on }[0, T)\},
$$

where a solution is understood in the sense of Definition 1.1. Let us first take care of uniqueness: Since any solution of problem (1.1) is of class $(\mathrm{mb})^{(3, \beta)}$, the uniqueness of time-local solutions implies that on some nontrivial interval $[0, \tau)$ there can only be one solution. Let us assume that there are two different maximal solutions, say $\left(v_{1}, p_{1}, \Gamma_{1}\right),\left(v_{2}, p_{2}, \Gamma_{2}\right)$. Then continuity implies that

$$
D:=\left\{t:\left(v_{1}, p_{1}, \Gamma_{1}\right)(t) \neq\left(v_{2}, p_{2}, \Gamma_{2}\right)(t)\right\}
$$

is an open subset of $\left[0, t^{+}\right)$. Thus $t_{*}:=\inf D \notin D$. But we can use $\left(v_{1}, p_{1}, \Gamma_{1}\right)\left(t_{*}\right)=$ $\left(v_{2}, p_{2}, \Gamma_{2}\right)\left(t_{*}\right)$ as initial value for a unique time-local solution, existing on, say, $\left[t_{*}, t_{*}+\tau^{\prime}\right)$. Thus, we can extend $\left(v_{1}, p_{1}, \Gamma_{1}\right)$ to a unique classical solution on $\left[0, t_{*}+\tau^{\prime}\right)$. But $\left[t_{*}, t_{*}+\tau^{\prime}\right) \cap D \neq \emptyset$, which is a contradiction.

Now, let $N(t)$ be a tubular neighborhood of $\Gamma(t)$ and suppose that there are constants $C, \varepsilon_{0}>0$ such that

(i) $\|v(t)\|_{B U C^{1}(\Omega(t))}+\|p(t)\|_{B U C^{1}(\Omega(t))} \leqslant C$,

(ii) $A(\Gamma(t)) \geqslant \varepsilon_{0}$.

Let us assume that $t^{+}$is finite. First of all we observe

$$
\left|\frac{\mathrm{d}}{\mathrm{d} t} \operatorname{area}(\Gamma(t))\right|=\left|\frac{\mathrm{d}}{\mathrm{d} t} \int_{\Gamma(t)} 1\right|=\left|\int_{\Gamma(t)} V(t) \cdot H(t)\right| \leqslant \frac{1}{\varepsilon_{0}} \cdot C \cdot \operatorname{area}(\Gamma(t)),
$$

i.e. $\sup _{t \in\left[0, t^{+}\right)}$area $(\Gamma(t))<\infty$, since $t^{+}<\infty$. Therefore, invoking Lemma 2.2, the family $\{\Gamma(t)\}$ has uniformly bounded $C^{2}$-geometry. We fix a smooth atlas for each surface $\Gamma(t)$ and a corresponding partition of unity as constructed in the proof of Lemma 2.2. In particular, we choose $m, K, L$ according to Lemma 2.2. Keep in mind that the norms of the function spaces built over $\Gamma(t)$ are fixed from now on. If now $t^{*} \in\left[0, t^{+}\right)$is given, then for some $\delta^{*}=\delta^{*}\left(t^{*}\right)>0$ the solution has a time-local representation $\left(u^{*}, \rho^{*}\right)$ in the form (3.6) on some interval $J\left(t^{*}\right):=\left[t^{*}, t^{*}+\delta^{*}\right)$, where $u_{0}^{*}=v\left(t^{*}\right)-\psi, \rho_{0}^{*}=0$ in the case $t^{*}>0$, since $\Gamma\left(t^{*}\right)$ is smooth. By (ii) we may assume that $r\left(N\left(t^{*}\right)\right)=\varepsilon_{0}$. For a technical reason (cf. Lemma 2.2) we choose $r\left(N\left(t^{*}\right)\right)=a_{0}$, where $0<a_{0}<\varepsilon_{0}$. Then $\left(u^{*}, \rho^{*}\right)$ is arranged to take values in the set

$$
\mathcal{U}\left(t^{*}\right):=H_{q, 0}^{2}\left(\Omega\left(t^{*}\right)\right) \times\left\{\rho \in B_{q q}^{4-1 / q}\left(\Gamma\left(t^{*}\right)\right):\|\rho\|_{c^{1+\beta}\left(\Gamma\left(t^{*}\right)\right)}<a_{0} / 5\right\},
$$


but our assumptions imply even stronger a priori bounds: Later on we will see that for some number $\tilde{\varepsilon}$,

$$
\sup _{t^{*} \in\left[0, t^{+}\right)} \sup _{x \in N\left(t^{*}\right), h \in J\left(t^{*}\right)}\left|\nabla\left(\varphi_{\rho^{*}(h)}\right)(x)\right| \leqslant \tilde{\varepsilon} .
$$

From this, $V=p_{v}$ and (i) we conclude

$$
\sup _{t^{*} \in\left[0, t^{+}\right) J\left(t^{*}\right)} \sup _{t} \mid<\infty
$$

meaning that $\left\|\rho^{*}(\cdot)\right\|_{C\left(J\left(t^{*}\right), C\left(\Gamma\left(t^{*}\right)\right)\right)}$ will vanish as $t^{*} \rightarrow t^{+}$. We will see at the very end of the proof that also the quantity

$$
\left\|\rho^{*}\right\|_{C\left(J\left(t^{*}\right), C^{1+\beta}\left(\Gamma\left(t^{*}\right)\right)\right)}
$$

will vanish as $t^{*} \rightarrow t^{+}$.

On the other hand, if $t^{*} \in\left[0, t^{+}\right)$is given, as in the proof of Lemma 3.2 the definition of the mapping $\sigma \mapsto \theta_{\sigma}$ immediately implies

$$
\sup _{\delta \in J\left(t^{*}\right)}\left(\left\|\theta_{\rho^{*}(\delta)}\right\|_{C^{1}\left(\overline{\Omega\left(t^{*}\right)}, \mathbb{R}^{n}\right)}+\left\|\theta_{\rho^{*}(\delta)}^{-1}\right\|_{C^{1}\left(\overline{\Omega\left(t^{*}+\delta\right)}, \mathbb{R}^{n}\right)}\right)<\infty .
$$

This estimate may depend on $t^{*}$. Nevertheless, $\left(r^{*}, u^{*}\right) \in L_{\infty}\left(J\left(t^{*}\right), B U C^{1}\left(\Omega\left(t^{*}\right)\right) \times\right.$ $\left.B U C^{1}\left(\Omega\left(t^{*}\right)\right)\right)$, and we can arrange the time-local solution $\left(u^{*}, \rho^{*}\right)$ to exist until it reaches the boundary of $\mathcal{U}\left(t^{*}\right)$, thanks to Lemma 3.2, contradicting (3.10).

It remains to prove (3.8) and (3.10). Fix $t^{*} \in\left(0, t^{+}\right)$and let $x \in N\left(t^{*}\right)$. Let $\left\{\left(W_{1}, \varphi_{1}^{-1}\right), \ldots,\left(W_{m_{x}}, \varphi_{m_{x}}^{-1}\right)\right\}$ be those charts of $\Gamma\left(t^{*}\right)$ that contain $P_{\left[\Gamma\left(t^{*}\right)\right]}(x)$, that is,

$$
P_{\left[\Gamma\left(t^{*}\right)\right]}(x) \in \bigcap_{l=1}^{m_{x}} W_{l} \cap \Gamma\left(t^{*}\right) .
$$

If $\left\{\pi_{j}: 1 \leqslant j \leqslant m\right\}$ is the corresponding subordinate partition of unity, and if $\operatorname{supp}\left(\pi_{l}\right) \subset W_{l} \cap$ $\Gamma\left(t^{*}\right)$ for $1 \leqslant l \leqslant m_{x}$, the assertion follows from the decomposition

$$
\rho^{*}(t) \circ P_{\left[\Gamma\left(t^{*}\right)\right]}=\left(\sum_{l=1}^{m_{x}} \pi_{l}\right) \rho^{*}(t) \circ \varphi_{l}^{-1} \circ \varphi_{l} \circ P_{\left[\Gamma\left(t^{*}\right)\right]}=\left[\sum_{l=1}^{m_{x}}\left(\pi_{l} \cdot \rho^{*}(t)\right) \circ \varphi_{l}^{-1}\right] \circ\left[\varphi_{l} \circ P_{\left[\Gamma\left(t^{*}\right)\right]}\right],
$$

due to the bounded geometry of $\{\Gamma(t)\}$, Lemma 2.2 and $\left\|\rho^{*}\right\|_{C\left(J\left(t^{*}\right) C^{1}\left(\Gamma\left(t^{*}\right)\right)\right)} \leqslant a_{0} / 5$. An estimate for $D P_{\left[\Gamma\left(t^{*}\right)\right]}$ in terms of the principal curvatures of $\Gamma\left(t^{*}\right)$ (dominated by $1 / \varepsilon_{0}$ ) can be obtained by inverting equation (14.97) in Chapter 14.6 of [GilTru].

In order to prove (3.10), let us economize our notation and drop $t^{*}, l$ unless it is necessary. Let $Y:=\varphi\left[\Gamma \cap \varphi^{-1}[W]\right] \subset \mathbb{R}^{n-1}$ and write again $\varphi^{-1}$ instead of $\left.\varphi^{-1}\right|_{Y}$. After possibly performing a suitable rotation and translation of coordinates, we may assume that $Y$ is a 0 -neighborhood in $\mathbb{R}^{n-1}$ as well as $\varphi^{-1}(x)=(x, g(x))$ in $Y$. A careful inspection of the proof of Lemma 2.2 shows that we may assume

- $\|\nabla g\|_{C(\bar{Y})} \leqslant 1$

- $Y=\mathbb{B}(0, R)$ for some $R>0$;

- $\bigcup_{l=1}^{m} \varphi^{-1}\left(l, t^{*}\right)\left[Y_{\varepsilon}\right]=\Gamma\left(t^{*}\right)$, where $Y_{\varepsilon}:=\mathbb{B}(0, R-\varepsilon), 0<\varepsilon<R$;

- $\pi$ is subordinate to $\varphi^{-1}\left[Y_{\varepsilon}\right]$. 
Then, in order to prove (3.10), it suffices to show that for some constant $\tilde{K}$,

$$
\left\|\rho^{*}(h) \circ \varphi^{-1}\left(l, t^{*}\right)\right\|_{W_{S}^{2}\left(Y_{\varepsilon}\right)} \leqslant \tilde{K}, \quad l \in\{1, \ldots, m\}, h \in J\left(t^{*}\right),
$$

where $\tilde{K}$ depends on the global constants $K, L, a_{0}$, and $s$ is large enough that $W_{s}^{2} \hookrightarrow c^{1+\gamma}$ where $\gamma>\beta$.

Let $G(x, z):=g(x)-z$ for $x \in Y$ and $z \in \mathbb{R},|z|<R$. Then $\varphi^{-1}[Y]=G^{-1}[\{0\}]$. Observe that the matrix

$$
a_{i j}(\zeta):=\delta_{i j}-\zeta_{i} \zeta_{j} /\left(1+|\zeta|^{2}\right), \quad \zeta \in \mathbb{R}^{n-1}
$$

is positive definite ( $\delta_{i j}$ being the Kronecker symbol). Thus, the operator $A$ defined by

$$
H(x, g(x))=\operatorname{div}\left(\frac{\nabla g(x)}{\sqrt{1+|\nabla g(x)|^{2}}}\right)=\frac{1}{|\nabla G|} \cdot \sum_{i, j=1}^{n-1} a_{i j}(\nabla g(x)) \partial_{i} \partial_{j} g(x)=: A g(x)
$$

is uniformly elliptic in $Y$. Moreover, $\max _{i, j}\left\|a_{i j}(\nabla g(x))\right\|_{C^{1}(\bar{Y})} \leqslant c, c=c(K)$, and

$$
\nabla A g=A(\nabla g)+B
$$

where $B=B\left(D^{2} g\right)$ is also uniformly bounded in terms of $K$. From the fourth equation in (1.1) and our assumption on $p$ we know that $\nabla[H \circ(x, g(x))]=[A \nabla g+B](x)$ is a priori bounded in terms of $K$ and $C$. Thus, elliptic theory implies that

$$
\left\|\partial_{k} g\right\|_{W_{s}^{2}\left(Y_{\varepsilon}\right)} \leqslant c, \quad c=c(K, n, C, \varepsilon, s), 1 \leqslant k \leqslant n-1 .
$$

Now, let $v=v\left(t^{*}\right)$ be the outward unit normal vector field of $\Gamma=\Gamma\left(t^{*}\right)$, and let $\hat{v}:=v \circ \varphi^{-1}$ and $\hat{\rho}:=\rho^{*}(h) \circ \varphi^{-1}$. Let $\hat{\mu}$ be the outward unit normal vector field of the surface $\theta_{\hat{\rho}}[\Gamma]$. Then $\left\|\hat{v}_{j}\right\|_{W_{s}^{2}\left(Y_{\varepsilon}\right)}$ is estimated from above in terms of $c=c(K, n, C, \varepsilon, s)$. Since $\left\|\rho^{*}(h)\right\|_{c^{1+\beta}(\Gamma)} \leqslant a_{0}$, interpolation shows that

$$
\left\|\rho^{*}(h) \circ \varphi^{-1}\left(t^{*}\right)\right\|_{C^{1}(\bar{Y})}
$$

will vanish as $t^{*}$ reaches $t^{+}$, thanks to (3.9). Observe

$$
0=\partial_{j} 1=\partial_{j}(\hat{v} \cdot \hat{v})=2 \partial_{j} \hat{v} \cdot \hat{v}
$$

and thus

$$
\left(\partial_{j}\left(\varphi^{-1}+\hat{\rho} \hat{v}\right)\right) \cdot \hat{v}=\partial_{j} \hat{\rho} .
$$

Therefore, since the left factor in the last equation is tangential to $\theta_{\hat{\rho}}[\Gamma]$, we may assume that $\hat{v} \cdot \hat{\mu}$ is close to 1 . As in the proof of Lemma 2.2 it can be seen that the coordinate representation of the second fundamental form of the surface $\theta_{\hat{\rho}}[\Gamma]$,

$$
\left[I I\left(\theta_{\hat{\rho}}[\Gamma]\right)\right]_{i j}:=\left(\partial_{i} \partial_{j} \varphi^{-1}+\partial_{i} \partial_{j} \hat{\rho} \hat{v}+\partial_{i} \hat{\rho} \partial_{j} \hat{v}+\partial_{j} \hat{\rho} \partial_{i} \hat{v}+\hat{\rho} \partial_{i} \partial_{j} \hat{v}\right) \cdot \hat{\mu},
$$

is estimated in terms of $\varepsilon_{0}$ and $K$. The desired estimate of $\partial_{i} \partial_{j} \hat{\rho}$ follows immediately. 
REMARK 3.4 We use the same notation as at the end of the last proof and let $\rho:=\rho^{*}(h)$. Moreover, $D(\hat{\rho})$ denotes the localized version of the operator $D, D \in\{P, B T P\}$, i.e. $(D(\rho) f) \circ \varphi^{-1}$ $=D(\hat{\rho})\left(f \circ \varphi^{-1}\right)$, where $f$ denotes a sufficiently regular function on $\Gamma\left(t^{*}\right)$. From [EsSi97a] we know that the operator $P(\hat{\rho})$ is elliptic in $x \in Y$ if $\hat{\rho}$ is a priori bounded in $C^{1}(\bar{Y})$ and the matrix $\left[w_{j k}(x)\right]^{-1}$ with

$$
w_{j k}(x):=\left[\partial_{j} \varphi^{-1} \cdot \partial_{k} \varphi^{-1}+\hat{\rho}(h)\left(\partial_{j} \hat{v} \cdot \partial_{k} \varphi^{-1}+\partial_{k} \hat{v} \cdot \partial_{j} \varphi^{-1}\right)+\hat{\rho}(h)^{2}\left(\partial_{j} \hat{v} \cdot \partial_{k} \hat{v}\right)\right](x)
$$

is positive definite (cf. the proof of Lemma 3.2 in [EsSi97a]). Thus, the generation property of $P(\rho)$ depends on a smallness assumption for $\rho$ which can be made uniformly with respect to a uniformly bounded $C^{2}$-geometry of the family $\{\Gamma(t)\}$. Basically, three facts guarantee the generation property of $B(\rho) T(\rho) P(\rho)$ for $\rho \in c^{2+\varepsilon}(\Gamma)$ :

(i) ellipticity of $P(\hat{\rho})$;

(ii) ellipticity of $A(\rho)$;

(iii) positivity of $\vec{b}(\rho) \cdot v$,

where $\vec{b}(\rho)$ defines the action of $B(\rho)$ by $B(\rho) w=\vec{b}(\rho) \cdot \nabla w$. Notice that the requirements (ii) and (iii) are automatically fullfilled if $\theta_{\rho}$ is a diffeomorphism - they do not need a further smallness assumption for $\rho$. A few more details about this are given in [Li2009].

The sufficiency of (i)-(iii) may be deduced from a careful study of Lemmata 5.1, 5.2 and estimates (5.6), (5.7) in [EsSi97a]. More details are given in [EsSi97b], [EsSi95]. That is why we do not have to worry about (i)-(iii) when choosing the number $a_{0}$ in the proof of the preceding theorem.

Proof of Corollary 1.3. First observe that $u=v \equiv c$. We have

$$
\begin{aligned}
\frac{\mathrm{d}}{\mathrm{d} t} \operatorname{vol}(t) & =\int_{\Gamma(t)} V(t) \mathrm{d} x=-\int_{\Gamma(t)} p_{v}(t, x) \mathrm{d} \sigma(x)=-\int_{\Omega(t)} \Delta p(t, x) \mathrm{d} x \\
& =\int_{\Omega(t)} f(v(t, x)) \mathrm{d} x=-\alpha_{0} \operatorname{vol}(\mathrm{t}) .
\end{aligned}
$$

Thus, if $t^{+}=\infty$, then $\operatorname{vol}(\Omega(t)) \rightarrow 0$ as $t \rightarrow \infty$, thus $A(\Gamma(t)) \rightarrow 0$ : Indeed, if not, there would be a ball $B \subset \bar{\Omega}(t)$ for all $t \in\left[0, t^{+}\right)$and $\operatorname{vol}(\Omega(t)) \geqslant \operatorname{vol}(B)$. Let us assume $t^{+}<\infty$, but $A(\Gamma(t)) \geqslant a_{0}$ and $\|\nabla p(t)\|_{C(\Gamma(t))} \leqslant C$. From the corresponding a priori bounds for $V$ and $H$ (and thus area $(\Gamma(t))$, since $\left.t^{+}<\infty\right)$ it can be deduced that then

$$
D M:=\sup _{t} \operatorname{diam}(\Omega(t))<\infty .
$$

Thus $|p(t, x)| \leqslant C_{1}\left(\alpha_{0}, a_{0}, D M\right)$ (cf. Theorem 3.7 in [GilTru]). Moreover, observe that $\partial_{i} p(t)$ is harmonic in $\Omega(t)$ for $i=1, \ldots, n$, meaning that

$$
\sup _{t}|\nabla p(t, x)| \leqslant C_{2}, \quad x \in \Omega(t),
$$

with $C_{2}=C_{2}(C, D M)$ (cf. again Theorem 3.7 in [GilTru]), contradicting Theorem 1.2. This completes the proof.

\section{Acknowledgements}

The author is grateful to Joachim Escher for several fruitful discussions. Moreover, it must be emphasized that the idea of the proof of Theorem 2.3 is due to Matthias Bergner. 


\section{REFERENCES}

[Ama93] Amann, H. Nonhomogeneous linear and quasilinear elliptic and parabolic boundary value problems. In: Function Spaces, Differential Operators and Nonlinear Analysis, H. J. Schmeisser and H. Triebel (eds.), Teubner, Stuttgart (1993), 9-126. Zbl 0810.35037 MR 1242579

[BF2003] BAZALIY, B. V., \& FRIEDMAN, A. A free boundary problem for an elliptic-parabolic system: application to a model of tumor growth. Comm. Partial Differential Equations 28 (2003), 517560. Zbl 1031.35151 MR 1976462

[BEL2011] Bergner, M., Escher, J., \& Lippoth, F.-M. On the blow up scenario for a class of parabolic moving boundary problems. Submitted.

[ByCh96] Byrne, H. M., \& Chaplain, M. A. J. Growth of necrotic tumors in the presence and absence of inhibitors. Math. Biosci. 135 (1996), 187-216. Zbl 0856.92010

[CrLoNi] Cristini, V., Lowengrub, J., \& Nie, Q. Nonlinear simulation of tumor growth. J. Math. Biol. 46 (2003), 191-224. Zbl 1023.92013 MR 1968541

[Es2004] EsChER, J. Classical solutions to a moving boundary problem for an elliptic-parabolic system. Interfaces Free Bound. 6 (2004), 175-193. Zbl 1057.35097 MR 2079602

[EsMa10] Escher, J., \& MATIOC, A. V. Radially symmetric growth of nonnecrotic tumors. Nonlinear Differential Equations Appl. 17 (2010), 1-20. Zbl 1193.34036

[EsSi95] Escher, J., \& Simonett, G. Maximal regularity for a free boundary problem. Nonlinear Differential Equations Appl. 2 (1995), 463-510. Z Zbl 0842.35083 MR 1356871

[EsSi97a] Escher, J., \& Simonett, G. Classical solutions for Hele-Shaw models with surface tension. Adv. Differential Equations 2 (1997), 619-642. Z Zbl 1023.35527 MR 1441859

[EsSi97b] EsCHER, J., \& SimONETT, G. Classical solutions of multidimensional Hele-Shaw models. SIAM J. Math. Anal. 28 (1997), 1028-1047. Zbl 0888.35142 MR 1466667

[FR1999] FRIEDMAn, A., \& REITICH, F. Analysis of a mathematical model for growth of tumor. J. Math. Biol. 38 (1999), 262-284. MR 1684873

[FR2001a] Friedman, A., \& REITICH, F. Symmetry-breaking bifurcation of analytic solutions to free boundary problems: an application to a model of tumor growth. Trans. Amer. Math. Soc. 353 (2001), 1587-1634. Zbl 0983.35019 MR 1806728

[FR2001b] Friedman, A., \& ReITICH, F. Nonlinear stability of quasi-static Stefan problems with surface tension: a continuation approach. Ann. Scuola Norm. Sup. Pisa 30 (2001), 341-403. Zbl 1072.35208 MR 1895715

[GilTru] Gilbarg, D., \& Trudinger, N. Elliptic Partial Differential Equations of Second Order. Springer, Berlin (2001). Z Zbl 1042.35002 MR 1814364

[Gr1972] Greenspan, H. P. Models for the growth of a solid tumor by diffusion. Stud. Appl. Math. 51 (1972), 317-340. Z Zbl 0257.92001

[Gr1956] GreEnspan, H. P. On the growth and stability of cell cultures and solid tumors. J. Theor. Biol. 56 (1976), 229-242. MR 0429164

[Kn2007] KNEISEL, C. Über das Stefan-Problem mit Oberflächenspannung und thermischer Unterkühlung. VDM Verlag Dr. Müller (2008).

[Li2009] LipPOTH, F.-M. Singular limits and maximally continued solutions of moving boundary problems. Phd. thesis (2009).

[Lu1995] LunARDI, A. Analytic Semigroups and Optimal Regularity in Parabolic Problems. Birkhäuser, Basel (1995). Z Zbl 0816.35001 MR 1329547

[TrI83] Triebel, H. Theory of Function Spaces I. Birkhäuser, Basel (1983).

[TrII92] Triebel, H. Theory of Function Spaces II. Birkhäuser, Basel (1992). Zbl 0763.46025 MR 1163193 